\title{
TIME DEPENDENCE OF ION-MIGRATION EFFECTS IN NiCr RESISTOR FILMS
}

\author{
U. SMITH† and R. HOFFMANN \\ Thin Film Electronics Department, BALZERS AG, FL-9496 Balzers, Liechtenstein
}

(Received November 4, 1977, in final form February 27, 1978)

\begin{abstract}
This paper reports the effects of ion-migration on $\mathrm{NiCr}$-resistor films sputtered on the substrates Corning 0211 and 7059. Graphs showing the influence of magnitude and polarity of the bias potential, of the separation between resistor and counter-electrode, and of reversal of bias polarity are given for Corning 0211 substrates at a temperature of $100^{\circ} \mathrm{C}$ and in a relative humidity of $0.7 \%$ for periods of time ranging up to 1000 hours. Results for resistors having a $\mathrm{NiCr}$-oxide layer between the film and the Corning 0211 substrate as well as for $\mathrm{NiCr}$ resistors on Corning 7059 substrate are also presented. Previous use of a bias-electrode at positive potential is shown to lead to a depletion-recovery effect. The physical interpretation of the experimental features is given in terms of a model involving anodic oxidation at the positive electrode and formation of a low conductivity deposit at the negative electrode.
\end{abstract}

\section{INTRODUCTION}

An effect well known to users of sodium-rich substrates for thin film circuits is the large change in resistance for resistors having potentials considerably different from those of other nearby parts of the circuit. Depending upon the geometry of the resistor, the change in resistance can also be caused by potential differences between different regions of the resistor itself. These changes, which are superimposed on the changes caused by the normal ageing processes in the resistors, are due to migration of ions present in the substrates, under the influence of the electric field. The ions are transported to the resistor where they react with the resistor material and cause the resistance of the film to change.

The effects of ion migration on thin-film resistors has been studied in several materials, e.g. tin oxide, ${ }^{2}$ tantalum nitride, ${ }^{3-5}$ nickel-chromium ${ }^{1,6,7,9}$ and tantalum. ${ }^{11,12}$ A related investigation of galvanic effects due to ionic diffusion between aluminum and gold electrodes should also be mentioned.

The purpose of the work described here was to study in more detail the time dependence of the ionmigration effects in the technologically important $\mathrm{NiCr}$-resistor-films. The emphasis in the investigation was on studying the influence of voltage magnitude and polarity, electrode separation, and of reversal of

$\dagger$ Present address: Sandvik AB, Coromant Factory, S-12612 Stockholm 42 Sweden. bias polarity in the case of an alkali-rich substrate where the effects are most pronounced. In addition experiments were made with a substrate having a lower alkali content and also with substrates having an oxide layer beneath the resistors.

\section{EXPERIMENTAL}

The films used in the experiments were sputtered $\mathrm{NiCr}$-films with a resistance of about $100 \mathrm{ohms}$ per square. The contacting areas consisted of a gold film with a layer of titanium between the gold and the $\mathrm{NiCr}$.

Two kinds of substrates having quite different concentrations of alkali ions were used in the experiments. The alkali-rich substrate was Corning 0211, an alkali-zinc borosilicate with approximately 6.2 wt $\% \mathrm{Na}_{2} \mathrm{O}$ and 6.9 wt $\% \mathrm{~K}_{2} \mathrm{O}^{8}$ The other substrate was Corning 7059, an alumino-borosilicate with about $0.1 \mathrm{wt} \% \mathrm{Na}_{2} \mathrm{O} .5$ In some of the experiments a $\mathrm{NiCr}$-oxide film was deposited between resistor and substrate.

The electric field for ion-migration was obtained by applying a voltage between the resistor and an auxiliary electrode, both located on the same side of the substrate. The resistor-electrode configuration is indicated in Figure 1 and was such that two resistors (R) could share one electrode (E). The resistor and electrode contacts $(\mathrm{C})$ are also shown in Figure 1. 

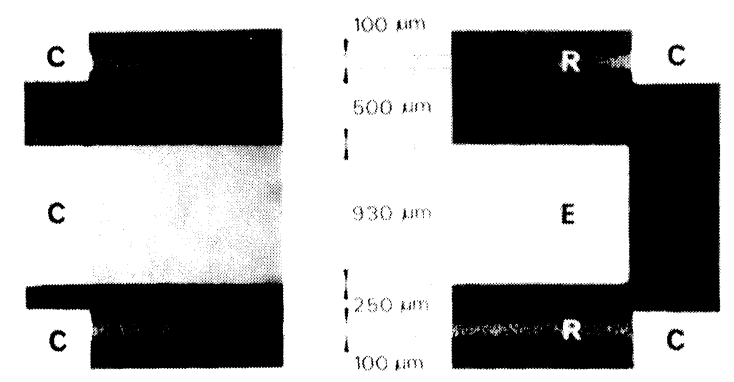

$-$

$18000 \mathrm{~mm}$

FIGURE 1 Configuration and dimensions of resistorelectrode system.

The electrode was made of the same material as the resistors i.e. $\mathrm{NiCr}$. The separations between resistors and electrode were $250 \mu \mathrm{m}$ and $500 \mu \mathrm{m}$ respectively. The resistor configuration was thus similar to that used in thin-film resistor networks. In order that the resistance change due to ion-migration could be separated from that of the regular ageing of the resistors, an additional resistor was located on the substrate a distance away from the resistor-electrode combination so as not to be influenced by the applied fields.

The resistors were biased at a constant potential relative to the electrode. They were kept at a temperature of $100^{\circ} \mathrm{C}$ and at the lowest relative humidity the climate cabinet would permit, i.e. 0.7 $\%$, throughout the experiment, including the time intervals when the resistance changes were being determined. The measurements were made with a bridge arrangement operating at $1 \mathrm{kHz}$. Since the high insulation-resistance of the substrates was part of the bias circuit, all leads to the substrates and all feed-throughs into the climate cabinet had to be insulated with Teflon so that no unintentional voltage division could take place.

The measured values for the resistance changes were normalized to the resistance values determined at the start of the experiment. The changes due to normal ageing were then subtracted from these relative values so that the effect of the applied field alone could be studied.

\section{RESULTS}

In addition to outlining the geometrical conditions as discussed above, Figure 1 shows the appearance of a pair of resistors on a Corning 0211 substrate after

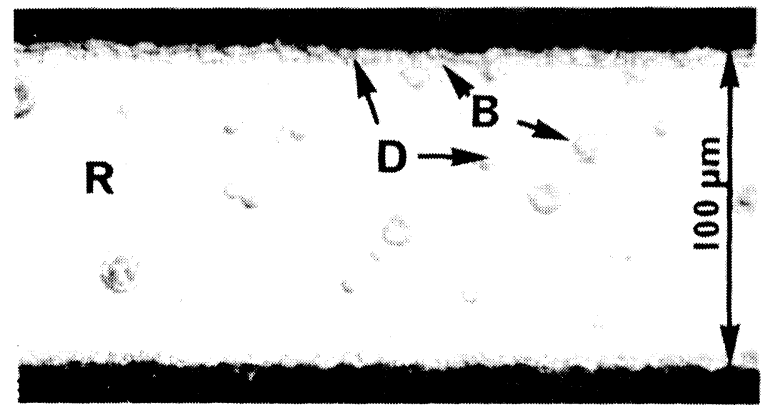

FIGURE 2 Appearance of a $\mathrm{NiCr}$ resistor on a Corning 0211 substrate after 356 hours at a bias of $-200 \mathrm{~V}$.

a negative potential of $200 \mathrm{~V}$ had been applied to the resistors with reference to the auxiliary electrode for a total of 935 hours. The severe attack on the resistors can be seen, whereas there are no changes to be seen on the electrode. The corresponding effect occurred for the opposite potential i.e. the resistors showed no change in appearance after having been subjected to a positive potential for the same length of time whereas the electrode had now been attacked in the same manner. In the latter case the severity of the attack tended to taper off towards the centre of the electrode surface. The effect was however irreversible: if the polarity was reversed half-way through the experiment the deterioration in the appearance of the resistor remained. In Figure 2 the nature of the attack is seen more clearly. Here, a resistor $(\mathrm{R})$ on a 0211 substrate is shown after having been subjected to a negative potential of $200 \mathrm{~V}$ for

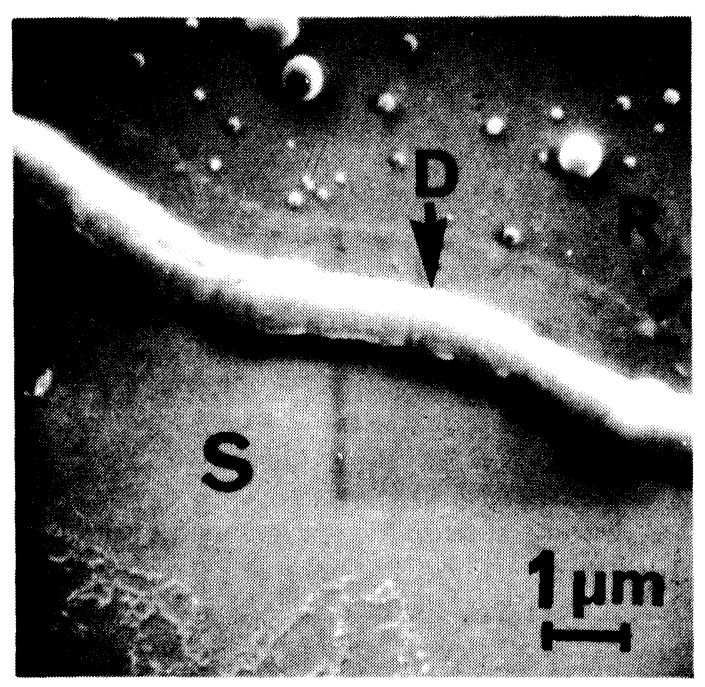

FIGURE 3 Scanning electron micrograph of a $\mathrm{NiCr}$ resistor after 56 hours at a bias of $-200 \mathrm{~V}$. 
a total of 356 hours. Heavy deposits (D) have formed along the edges and have also penetrated the film itself. The immediate surroundings (B) of the deposits appear brownish when viewed in an optical microscope. In addition to the heavy deposits, the resistors are also covered with small spherical spots. The latter spots and the heavy deposits (D) along the edge of the resistor can be seen more clearly in the scanning electron micrograph, Figure 3. The resistor (R) in Figure 3 with its Corning 0211 substrate (S) was biased at $-200 \mathrm{~V}$ for a total of 56 hours. After an experiment with a pair of resistors at negative potential the area densities of heavy deposits appeared to be equal on the two resistors surrounding one electrode despite the different resistor-electrode separations. The area densities of small spots were quite different for the two resistors, however. The density of small spots was much larger on the resistor which was farthest away from the electrode although one would expect the field strength, even though it is in homogeneous, to be considerably weaker at a separation of $500 \mu \mathrm{m}$ than at $250 \mu \mathrm{m}$. The reason for the high density is most likely that in the case of the more remote element, there is a greater volume of material available from which ions can be swept out.

Figure 3 also shows that a region of the substrate extending to about $5 \mu \mathrm{m}$ from the edge of the resistor was covered with some kind of deposit.

A pair of resistors which had been connected to $-140 \mathrm{~V}$ for 935 hours and had large amounts of deposits were rinsed in deionized water. All deposits and brownish discolourations of the type mentioned above present on the resistor surfaces before the rinse were thereby completely removed. However the deposit along the edge of the resistor which was not located on the resistor surface but on the substrate remained. No damage to the resistor film, e.g. in the form of holes caused by the deposits piercing the film, could be detected under microscopic examination. The resistance was measured before and after the resistors had been rinsed. The relative change in resistance was $+0.07 \%$ which is negligible in comparison with the resistance change due to ion-migration which amounted to $-1.65 \%$ over the 935 hours that the resistors were connected to $-104 \mathrm{~V}$.

An X-ray analysis of the deposit along the edges of the resistor in Figure 3 indicated the presence of sodium and chlorine. Other elements, namely silicon, potassium, titanium, aluminum and zinc, as well as nickel and chromium were also observed, but the origin of the signals for these elements was ascribed

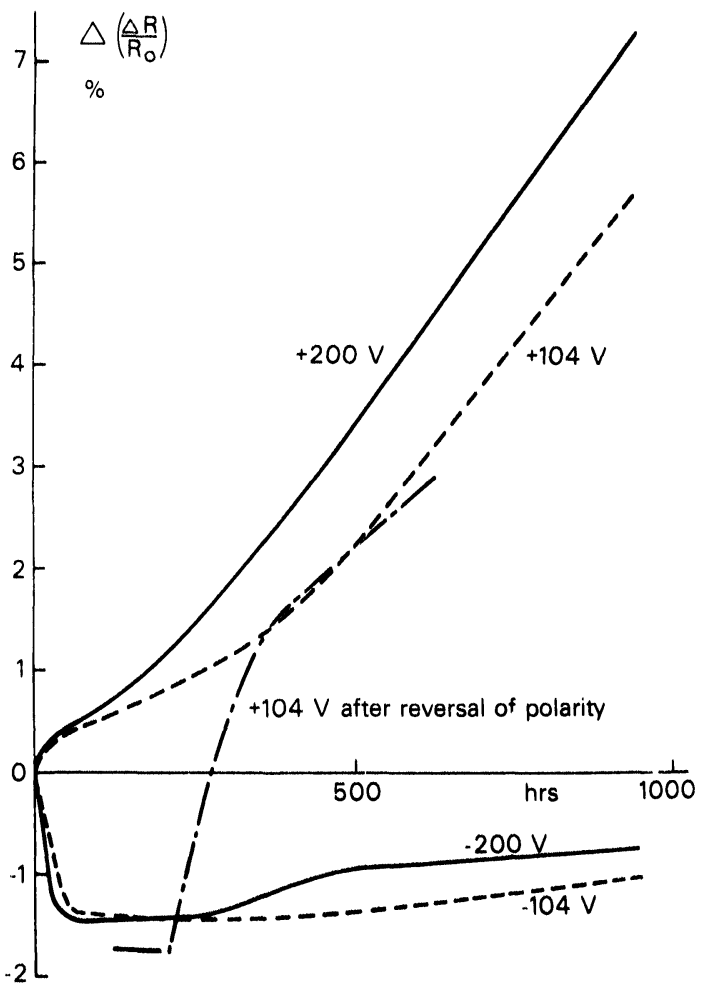

FIGURE 4 Resistance change with time for $\pm 200 \mathrm{~V}$ and $\pm 104 \mathrm{~V}$ bias.

to the penetration into substrate and resistor film, respectively of the electrons used to excite the $x$-rays. In particular, the sodium signal varied significantly between deposit and substrate, especially at lower accelerating voltages, but this was not seen to be the case with the potassium signal.

The dependence of the resistance change on time and voltage in the case of resistors on Corning 0211 substrates is shown in Figure 4. The curves show the relative change in resistance when the effects due to normal ageing have been subtracted. In addition to the curves for resistors at the potentials $\pm 200 \mathrm{~V}$ and $\pm 104 \mathrm{~V}$, respectively, a curve is also presented for a resistor where the bias was reversed after having been at $-104 \mathrm{~V}$ for 213 hours.

The dependence of the resistance change on resistor-electrode separation is shown in Figure 5. The resistors which had been deposited on a Corning 0211 substrate, were biased at $-104 \mathrm{~V},-56 \mathrm{~V}$ and $-32 \mathrm{~V}$ for a total of 213 hours. The curves show the resistance changes for the two electrode separations $250 \mu \mathrm{m}$ and $500 \mu \mathrm{m}$, respectively.

Measurements were also made on Corning 0211 substrates having a $380 \AA$ thick layer of $\mathrm{NiCr}$-oxide 


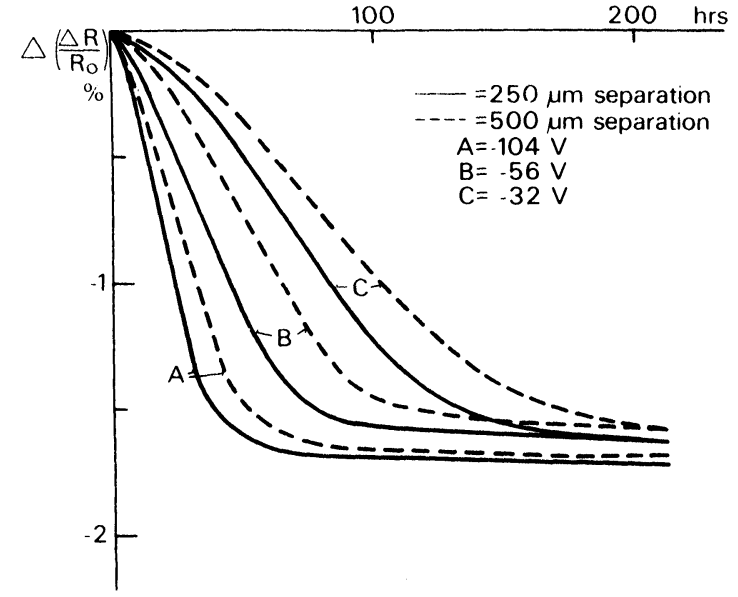

FIGURE 5 Resistance change with time for various negative bias-voltages and resistor-electrode separations.

between the resistor and the substrate. The magnitudes of the resistance changes were drastically reduced by the additional layer, the change for +200 $\mathrm{V}$ and $250 \mu \mathrm{m}$ seperation being about $150 \mathrm{ppm}$ after 200 hours and that for $-200 \mathrm{~V}$ about $750 \mathrm{ppm}$ after the same time. The curves for the positive potential increased almost linearly with time, whereas those for negative potentials were curved in the direction of an accelerating process in the same manner as the initial part of the curves in Figure 5.

In the case of resistors deposited on Corning 7059 substrates, the resistance changes showed an essentially linear time-dependence for times over 100 hours. Unlike the results for Corning 0211 substrates, the resistance changes were positive for both polarities. After a period of 1100 hours the total increase in relative resistance due to the electric field was $+650 \mathrm{ppm}$ for the resistors at $+200 \mathrm{~V}$ and 250 $\mu \mathrm{m}$ separation, and $+250 \mathrm{ppm}$ for those at $-200 \mathrm{~V}$. The increases during the initial $100 \mathrm{hrs}$ were 400 $\mathrm{ppm}$ for $+200 \mathrm{~V}$ and $50 \mathrm{ppm}$ for $-200 \mathrm{~V}$ bias. No tendency towards a saturation similar to that occurring after about 30 hours for negatively biased resistors on Corning 0211 substrates could be observed during the period of 1100 hours.

One experiment, exploring the nature of the reactions occurring at the resistors, took advantage of the fact that two resistors on a substrate shared one electrode as shown in Figure 1. Firstly, the resistor $500 \mu \mathrm{m}$ away from the electrode was connected to a potential of $-200 \mathrm{~V}$ relative to that of the electrode, while the other resistor was left at a floating potential. After about 100 hours, when the resistance change had reached well into the region of saturation at

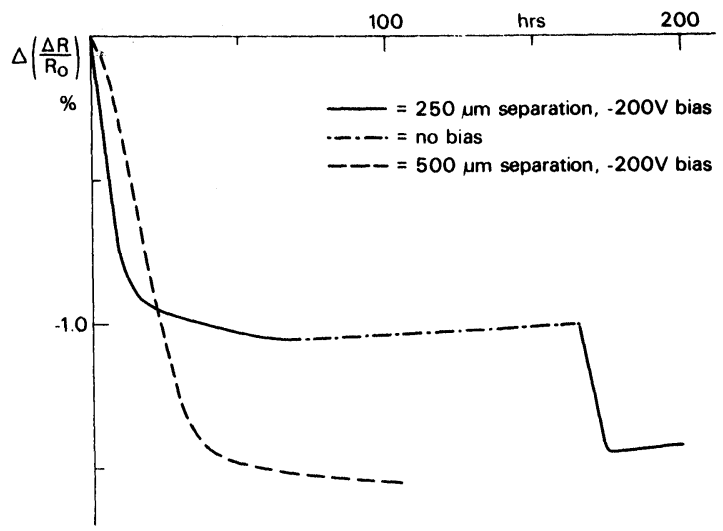

FIGURE 6 Resistance change for $-200 \mathrm{~V}$ bias with an intermediate recovery period.

about $-1.5 \%$, as the result in Figure 6 shows, the resistor at $250 \mu \mathrm{m}$ separation was connected to -200 $\mathrm{V}$ and the first resistor left floating. The resistance change for the resistor now at $-200 \mathrm{~V}$ commenced normally, but after about 5 hours the change decreased and shortly after saturated at about $-1.0 \%$, Figure 6. After the resistor had been in the saturation region for some time the bias was removed. The resistor-electrode system was then left to recover for about 95 hours. When the resistor was again biased at $-200 \mathrm{~V}$ the resistance changed at the original rate and saturated at a level comparable to that of the first resistor at $500 \mu \mathrm{m}$ separation, Figure 6.

\section{DISCUSSION}

Generally speaking, the increase in resistance for positive bias of the resistors with respect to the auxiliary electrode is attributed to a decrease in the thickness of the resistor film caused by an anodic oxidation $^{6}$ of the film material. The decrease in resistance for negative bias, on the other hand, is ascribed to the formation of low-conductivity reaction products at the interface between film and substrate. The details of the experimental results described above can be explained within the framework of this model as will be clear from the following discussion.

The lack of significant amounts of potassium in the deposit formed on negatively biased resistors is consistent with the assumption made by Kobale ${ }^{5}$ that primarily the sodium ions participate in the migration of ions through the substrate due to the relatively large size of the potassium ions. The 
presence of chlorine in the deposit appears to have its origin in the etchants used to produce the circuits for the ion-migration experiments. Unfortunately, the effects of electron-beam penetration do not allow any further conclusions to be drawn regarding the chemical composition of the deposit. The lack of change in resistance when the deposit had been dissolved away in deionized water is either due to an increase in resistance of the deposit when, after having penetrated the film, it is exposed to any moisture still present in the climate cabinet or to a negligible area of electrical contact between the deposit on top of the film and the film itself.

The observation that films with large amounts of deposit after a prolonged period at negative bias, Figures 1-3, showed no signs of mechanical damage is somewhat surprising in view of the amount of deposit formed. However, it is consistent with the behaviour of the resistance for negative bias, Figure 4 , since any major mechanical damage to the film caused by large amounts of deposit being pushed through the film should have lead to increases in resistance rather than the observed steady decrease. The deposit must therefore have been transported through the film via the metal grain boundaries. But, the tendency for the negative bias curves to bend over slightly in the positive direction after prolonged periods of time at saturation may be an indication that, over longer periods of time, the film does suffer damage to some extent. This would also explain the somewhat random character of the positive deviations illustrated by the behaviour of the two negative branches in Figure 4 after about 250 hours.

A gradual build-up of relatively low-conductivity reaction products along the interface of negatively biased resistors will also explain the saturation of the resistance change. Thus, when reaction products are added to the layer already formed, the effect on the resistance change will be progressively less as the thickness increases due to the low conductivity. However, the ultimate value for the resistance change cannot easily be calculated in the case of the present resistor-electrode configuration since it will be a function not only of the resistivity of the reaction products and the geometry of the resistors, but also of the distribution of the electric fields. In the present configuration this should be very inhomogeneous since the whole circuit is on one side of the substrate.

Although the sodium content in the substrate is very high, a depletion of ions could still occur in the form of a thin sheath around the electrode. The result shown in Figure 6 is believed to be due to such a depletion. Thus, it would seem that the period of negative bias for the first resistor has reduced the amount of sodium available to the second resistor so that a period of recovery is needed before the resistance change can reach the saturation level discussed above..

It is interesting to note that the initial behaviour of the curves in Figure 5 is consistent with a surface layer with lower sodium concentration than the bulk of the substrate. A reasonable order of magnitude for the thickness of this layer is obtained when relating the applied voltages to the times required for the resistance change in Figure 5 to reach the inflexion point. These times are taken to correspond to the times it takes for the ions to cross the surface layers at the various bias voltages. An estimate of the width of the surface layer is then obtained from the field strength and the mobility. Also the accelerating decrease in resistance observed for Corning 0211 substrates covered with a layer of $\mathrm{NiCr}$-oxide is considered to be caused by the oxide layer behaving as a zone initially depleted from sodium. This implies that the oxide layer will only provide a temporary protection against the effects of sodium migration.

The rapid increase in resistance which occurs when the polarity of the resistor bias is changed from negative to positive, Figure 4, appears to be due to a dissociation of reaction products formed during the period at negative bias. Also, the initial part of the resistance changes for positive bias voltages in Figure 4 , have the same curvature as the curve for the reversed bias. This could be due to a dissociation of the same kind since a certain amount of reaction products should have formed during the deposition of the film by a reaction between the film material closest to the substrate and the sodium present at the site of the deposition.

Since the long-term resistance changes for positive bias are larger than those for negative bias it is understandable that the results for resistors under load are reported to show an increase in resistance, ${ }^{3,6,7}$ the increase being the net result of the reactions taking place at the high-and low-potential regions of the resistor, respectively.

The behaviour of the biased NiCr-films is different from that of the tin-oxide films reported by $\mathrm{Cox}^{2}$ The resistance changes of the $\mathrm{NiCr}$-films are considerably more asymmetric than those of the tin-oxide films even when compared to the case where the presence of moisture was destroying the symmetry of the test geometry. Thus, in contrast to the result for the tin-oxide films, the over-all curvature for the resistance changes in Figure 4 has 
the same sign for both polarities. Furthermore, the curve for positive bias shows no sign of saturation. Moreover, the transition to saturation for negative bias is more abrupt for $\mathrm{NiCr}$. This leads to the conclusion that the shape of the curves is not only dictated by the transport properties of sodium in the substrate and the interface region which have been analyzed by $\mathrm{Cox},{ }^{2}$ but also by the specific nature of the chemical reactions occurring at the resistor and the electrode due to the electric field.

The present results show that, in addition to a reduction in the alkali content of the substrate, the introduction of an oxide layer between film and substrate can reduce the effects of ion-migration even in the case of such alkali-rich substrates as Corning 0211 . However, due attention must be paid to the fact that the oxide layer only provides a delaying action.

In addition to furthering the understanding of the ion-migration effects in the case of $\mathrm{NiCr}$-film resistors, the present results emphasize the importance of including the effects of electric fields over sufficiently long periods of time when evaluating the stability of resistor films.

\section{ACKNOWLEDGEMENTS}

The authors would like to thank Dr. G. Zinsmeister for suggesting the problem and for many stimulating discussions on the subject.

Special thanks are due to Dr. J. T. Law for valuable discussions of the experimental results as well as the final manuscript and to Drs. G. Sele and B. Solly for their thorough reading of the manuscript.

\section{REFERENCES}

1. G. Siddall and B. A. Probyn, Vacuum deposited metal film resistors, Brit. J. Appl. Phys., 12, 668 (1961).

2. S. M. Cox, Ion migration in glass substrates for electronic components, Phys. Chem. Glasses, 5, 161 (1964).

3. D. A. McLean, N. Schwartz and E. D. Tidd, Tantalumfilm technology, Proc. I.E.E.E., 52, 1450 (1964).

4. W. H. Orr and A. J. Masessa, The effect of substrate ion migration on thin film tantalum nitride resistors, $J$. Electrochem. Soc., 112, 1836 (1965).

5. R. W. Berry, P. M. Hall and M. T. Harris, Thin Film Technology (Van Nostrand Reinhold Co., New York, 1968), p. 408.

6. M. Kobale, Glas als Substrat, Nachrichtentechnische zeitschrift Nr. 10, 528 (1970).

7. Y. Nishimura, A. Nagata and H. Sasaki, Influence of Te and Tp values on the stability of film resistors. Fujitsu Sci. Techn. J., 3, 167 (1967).

8. L. I. Maissel and R. Glang, etd., Handbook of Thin Film Technology (McGraw-Hill Book Co., 1970), p. 6-8.

9. P. C. Smith and M. Genser, Investigation of thin film resistor failure, Physics of Failure in Electronics, 3, 306 (1964).

10. J. J. Wortman and R. M. Burger, Galvano-diffusion effect in thin film metal dielectric structures, Physics of Failure in Electronics, 3, 333 (1964).

11. S. S. Choi, Radiochemical study of lateral ion migration in insulting substrates for thin film microcircuits, Physics of Failure in Electronics, 4, 408 (1966).

12. M. Walker et al, Accelerated ageing and failure mechanism analysis of thin tantalum film $R-C$ networks, Physics of Failure in Electronics, 4, 179 (1966). 

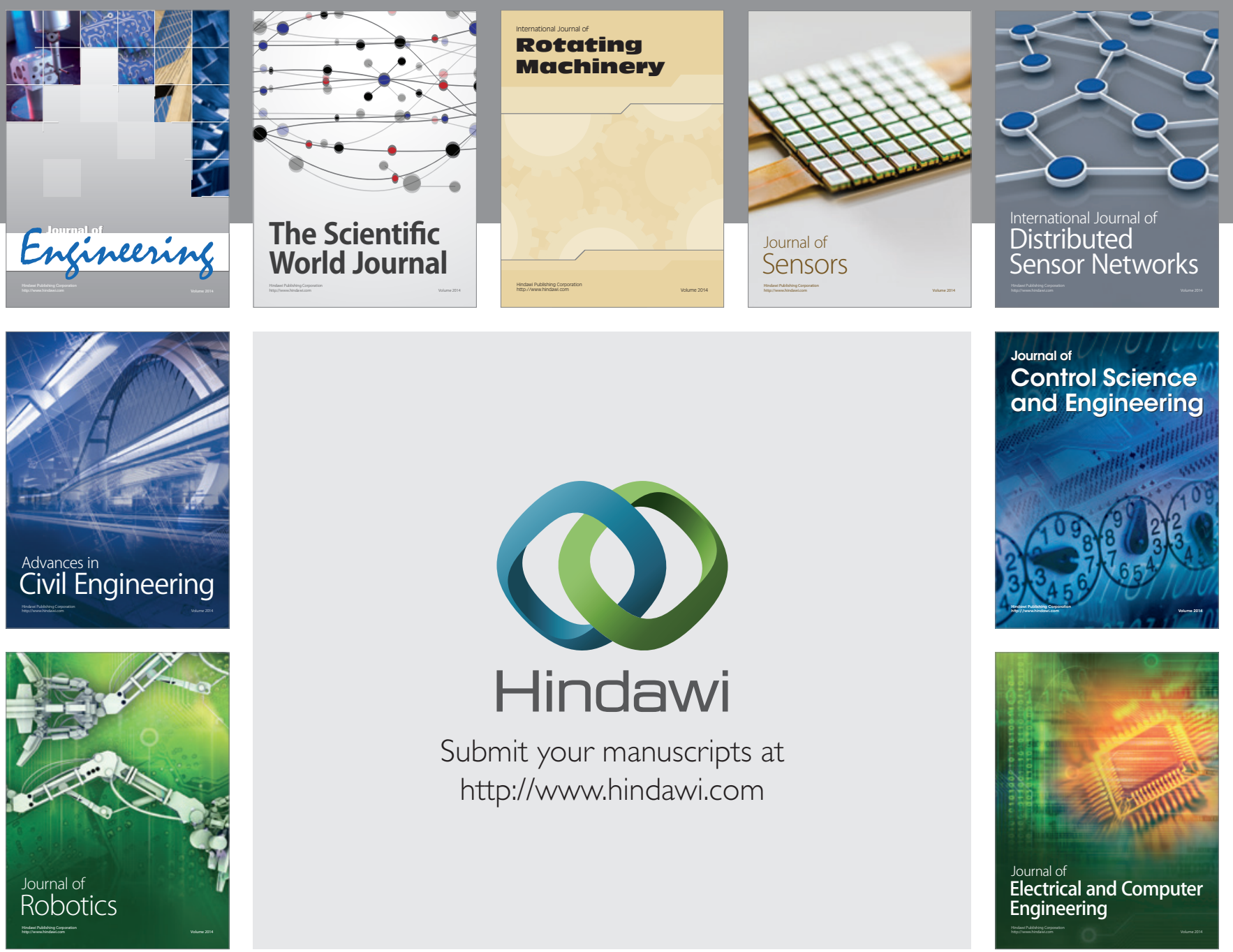

Submit your manuscripts at

http://www.hindawi.com
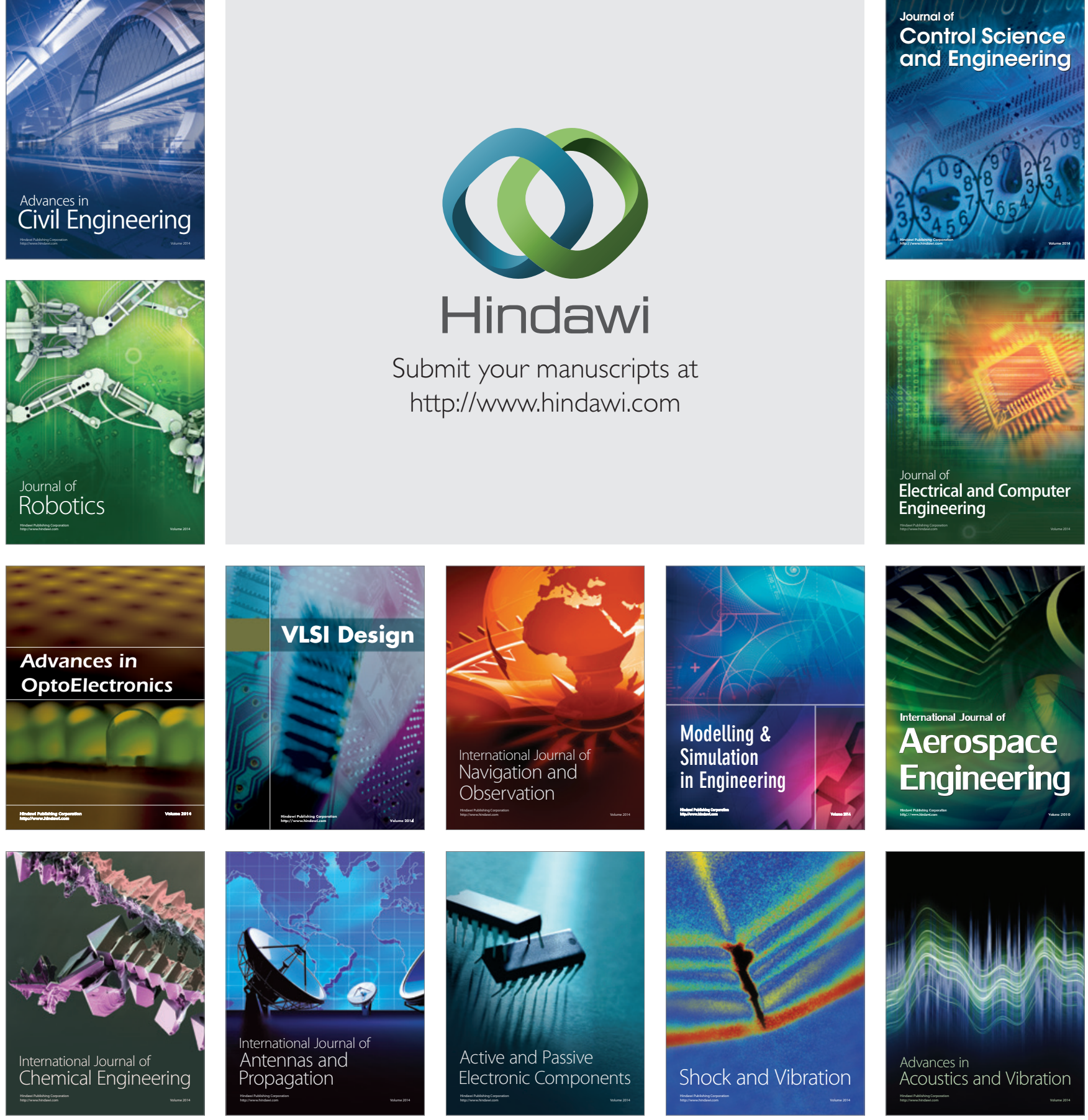